\title{
Electrochemical Dyeing of Cotton Fiber for Environmental Protection
}

\author{
Wei XIONG ${ }^{\mathrm{a}, 1}$, Yuxin YANG ${ }^{\mathrm{a}}$, Yinyan HOU ${ }^{\mathrm{a}}$, Guinian $\mathrm{HUANG}^{\mathrm{a}}, \mathrm{He} \mathrm{Li}^{\mathrm{a}}$ and Gang \\ $\mathrm{GAN}^{\mathrm{b}}$ \\ ${ }^{a}$ Hubei Key Laboratory of Biomass Fiber and Eco-dyeing \& Finishing, College of \\ Chemistry and Chemical Engineering, Wuhan Textile University, Wuhan, China \\ ${ }^{\mathrm{b}}$ Hubei Key Laboratory of Biomass Fiber and Eco-dyeing \& Finishing, College of \\ Physical Culture, Wuhan Textile University, Wuhan, China
}

\begin{abstract}
For environment-friendly dyeing, electrochemical dyeing method was researched. The low consumption of additives during dyeing is beneficial to environmental protection, and the electric field of electrochemical dyeing reduces the salt. Moreover, the K/S value of the fabric increases significantly at the same dye concentration. The alkaline electrolyte in the electrolytic cell may enhance the self-corrosion of the electrode during the electrochemical dyeing process.The results show that the electrochemical dyeing can reduce the amount of additives during the dyeing process.
\end{abstract}

Keywords. Electrochemical dyeing, environment-friendly dyeing, dyes, electrochemical treatment

\section{Introduction}

In order to solve the problems of water consumption, energy consumption, high chroma and pollution of reactive dyeing of cotton fabric, it is necessary to carry out energy-saving innovative design for reactive dye dyeing of cotton fabric. The realization of low alkali dyeing and the reduction of wastewater discharge and pollution are the key issues concerned by textile printing and dyeing workers at home and abroad. In recent years, salt free / low salt dyeing of reactive dyes has become a hot spot in dyeing and finishing industry.

The key of traditional salt free dyeing is how to eliminate the electrostatic repulsion between dye and fiber, and realize the efficient covalent combination between dye and fiber. It mainly includes the following ways [1]: converting the electrostatic repulsion between the fiber and the dye into electrostatic attraction; synthesizing cationic reactive dyes; using a medium that can make the fiber and / or dye charged [2-10].

However, due to the complex operation process, poor levelness, high cost and other shortcomings, these technologies can only be explored in the laboratory, but far from the large-scale use of industrialization. The electrochemical salt free dyeing

\footnotetext{
${ }^{1}$ Wei Xiong, Hubei Key Laboratory of Biomass Fiber and Eco-dyeing \& Finishing, College of Chemistry and Chemical Engineering, Wuhan Textile University, Wuhan, China; E-mail: wxiongid@wtu.edu.cn.
} 
technology has many advantages, such as easy control, mild reaction conditions, little damage to fiber, energy saving and emission reduction, simple operation, excellent dyeing effect and environment-friendly [11-20].

The principle of electrochemical salt free dyeing with reactive dyes is that positive charges are generated in the anode region under the electric field system, which can eliminate the repulsion of negative charges on the surface of cotton fabrics to the anion groups of reactive dyes, and promote the enrichment of dye anions in the anode region. This method is conducive to the full contact between the dye and the fabric in the anode area, so as to improve the dye uptake rate, abandon the use of salt and reduce environmental pollution. As seen in Fig. 1, electrochemical methods can effectively control the dyeing process and effect [21-28].

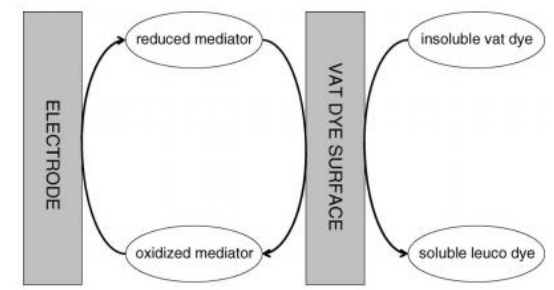

Figure 1. Principle of indirect electrochemical reduction system.

In recent years, some domestic dyeing workers are also engaged in the research of electrochemical reduction dyeing. Zang Guoqiang et al. Studied the electrochemical dyeing process of kn type reactive dyes with different structures. The $\mathrm{K} / \mathrm{S}$ value of the dyed fabric was improved, the color fastness was basically the same, the breaking strength of the fabric was improved, and the COD of the dye residue was significantly reduced. Pan Jie et al. Studied the electrochemical dyeing process of K-type reactive dyes with different structures. It was found that under the condition of electrochemical salt free dyeing, the larger the proportion of sulfonic groups in reactive dyes, the higher the $\mathrm{K} / \mathrm{S}$ value after dyeing. Wang Haiying et al. Have studied the electrochemical salt free dyeing of reactive dyes. The results show that the electrochemical salt free dyeing can significantly increase the dye uptake when dyeing cotton fabric with reactive dyes by dip dyeing. Cai Xinbin et al. Studied the electrochemical salt free dyeing process of reactive brilliant blue B-RV and obtained good results. But up to now, most of the researches are still on the dyeing process, and the follow-up more in-depth research is less.

Reactive dyeing technology has developed rapidly, and has made a lot of contributions to the development of salt free dyeing technology at home and abroad. Compared with the traditional salt dyeing, salt free dyeing is undoubtedly one of the requirements of sustainable development, but also brings substantial benefits to the people, which is also the direction of future printing and dyeing workers.

However, the disadvantages can not be ignored. The levelness and color fastness of cotton fabrics need to be strengthened, and the breaking strength of cotton fabrics should be further strengthened. In order to better promote the dyeing of reactive dyes, the structure of reactive dyes should be designed, and at the same time, the environmental protection oriented dyeing technology should be inclined, and the matching dyeing auxiliaries should be developed. More clean, efficient, environmental protection, more comfortable, better quality reactive dyeing process will be improved. 
This experiment focuses on the possibility of application. The application of electrochemical salt free dyeing in reactive dyeing was studied. In order to further explore the feasibility of this process, the dyeing performance by different additive reagent in dyeing process was studied.

\section{Experimental}

Dyeing was carried out in an electrolytic cell. The cotton was placed in the anode area and applied with different voltage. In addition, the electrochemical performance of the system was tested in the electrochemical workstation.

\subsection{Results and Discussion}

\subsubsection{Addives}

Since the electric field can replace the salt in traditional dyeing, the amount of salt can be greatly reduced by electrochemical dyeing. By providing an electric field to the dye liquor, the dye anions migrate to the anode in the dyeing bath. The cotton fabric to be dyed is connected with the positive electrode, which increases the dye anion concentration near the fabric, promotes the dye adsorption and improves the dye uptake.

In addition, the external electric field can increase the chemical sites of dyes in the dyeing solution and decrease the chemical sites in the fibers, which will promote the transfer of dyes from liquid phase to solid phase, increase the diffusion coefficient of dyes, and increase the K/S value. the cotton with electrochemical dyeing method shows better performance

\subsection{2. $\mathrm{K} / \mathrm{S}$}

Compared with the traditional dyeing method, the $\mathrm{K} / \mathrm{S}$ value of the electrochemical dyeing method is significantly increased. However, when the applied current is too high, on the one hand, the rate of water electrolysis increases at the same time; on the other hand, with the increase of the current density of the whole electric field, the amount of dye in the anode area increases, and the hydrophobic part of some dyes molecules passes through hydrogen bonds and The effect of van der Waals force makes the concentration of dyes decrease in the whole dyeing bath. Therefore, if the electric field intensity is too large, the $\mathrm{K} / \mathrm{S}$ value will also decrease.

The K/S value of the fabric increases significantly at the same dye concentration. As shown in figure 2 and figure 3, the cotton with electrochemical dyeing method shows good performance of dyeing.

The cotton with electrochemical dyeing has more uniform staining than that with traditional dyeing. The red and blue samples with electrochemical dyeing have deeper color than hat with traditional dyeing. Moreover, the amount of dyestuff required by electrochemical dyeing method may be reduced for fabrics with the same dyeing effect. 

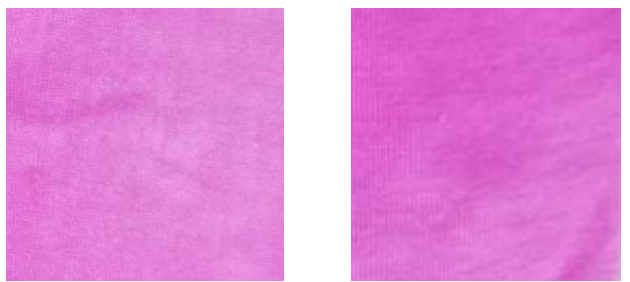

Figure 2. The red of traditional dyeing(left), and electrochemical dyeing(right).
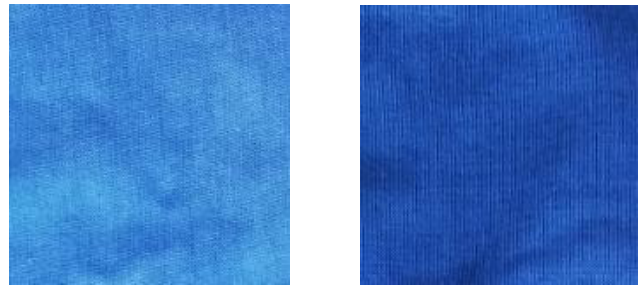

Figure 3. The blue of traditional dyeing(left), and electrochemical dyeing(right).

\subsubsection{Reduction of Dyeing}

The dye uptake rate is equivalent to the desorption rate because the dye and fiber gradually form an adsorption equilibrium. On the other hand, the dye anion concentration in the dye solution decreases continuously, and the dye anion adsorbed on the fabric surface has a reverse movement under the influence of anode region.

As a result, the concentration of dye anions on the fabric surface is reduced.The electric field of electrochemical dyeing can increase the dye uptake rate, so it can reduce the dyeing time.

At the same time, the fixation effect is better and the fixation time is shorter. On the one hand, the addition of alkali agent improves the $\mathrm{pH}$ value of dyeing solution, which makes the hydroxyl groups of cellulose more easily dissociate into anions, breaks the adsorption equilibrium between dyes and fibers, and slows the desorption rate on the fibers, so that some dyes can dye on the fibers; on the other hand, under the electrochemical action, sodium carbonate as a strong electrolyte greatly improves the electric field strength Traction is also greatly improved. However, with the prolongation of fixation time, the increase of $\mathrm{K} / \mathrm{S}$ value of electrochemical salt free dyeing fabric decreases.

\subsubsection{Microstructure.}

The microstructure of electrode after dyeing in the electrolytic cell may have serious corrosion because of the alkaline electrolyte.

\section{Conclusion}

The electrochemical dyeing method can reduce the amount of additives during the dyeing process. Meanwhile, the external electric field can increase the chemical sites of dyes in the dyeing solution and decrease the chemical sites in the fibers. The addition of alkali agent improves the $\mathrm{pH}$ value of dyeing solution. 


\section{Acknowledgments}

This work is supported by the Foundation of Hubei Provincial Education Department (Q20131607).

\section{References}

[1] Lee J, Kwon H, Seo J, et al. Conductive fiber-Based ultrasensitive textile pressure sensor for wearable electronics. Advanced Materials. 2015 Feb; 27(15): 2433-2439.2.

[2] Mu BN, Liu LY, Li W, et al. High sorption of reactive dyes onto cotton controlled by chemical potential gradient for reduction of dyeing effluents. Journal of Environmental Management. 2019 Jan; 239: 271-278.

[3] An Y, Ma JR, Zhu ZX, et al. Study on a water-saving and salt-free reactive dyeing of cotton fabrics in non-aqueous medium of liquid paraffin system. The Journal of The Textile Institute. 2020 Jan; 111 (10): 1538-1545.

[4] Ryou MH, Lee YM, Park JK, et al. Mussel-inspired polydopamine-treated polyethylene separators for high-power Li-ion batteries. Advanced Materials.2011 May; 23 (27): 3066-3070.

[5] Niu TJ, Wang XM, Wu CH, et al. Chemical modification of cotton fabrics by a bifunctional cationic polymer for salt-free reactive dyeing .ACS Omega.2020 Jun; 5 (25): 15409-15416.

[6] Tansil NC, Koh LD. Han MY. Functional silk: Colored and luminescent. Advanced Materials.2012 Feb; 24 (11): 1388-1397.

[7] Anis P, Toprak T and Kutlu E. Sericin assisted eco-friendly reactive dyeing for cotton fabric. Cellulose.2019 May; 26 (10): 6317-6331.

[8] Shin M, Song JH, Lim GH, et al. Highly stretchable polymer transistors consisting entirely of stretchable device components. Advanced Materials.2014 Mar; 26 (22): 3706-3711.

[9] $\mathrm{Mu} \mathrm{BN}, \mathrm{Li} \mathrm{W}, \mathrm{Xu} \mathrm{HL}$, et al. Salt-free and environment-friendly reactive dyeing of cotton in cottonseed oil/water system.. Cellulose. 2019 May; 26 (10): 6379-6391.

[10] Arivithamani N and Rengaswami V. Characterization and comparison of salt-free reactive dyed cationized cotton hosiery fabrics with that of conventional dyed cotton fabrics. Journal of Cleaner Production. 2018 May; 183: 579-589.

[11] Wang KK, Li XY, Yao JM. Effect of different iron salt media on indigo electrochemical reduction system. Fangzhi Xuebao/Journal of Textile Research. 2020 Jan; 41(1).

[12] Khandegar V, Saroha A K. Electrochemical Treatment of Effluent from Small-Scale Dyeing Unit. Indian Chemical Engineer. 2013 Jun; 55(2): 112-120.

[13] Wang Z, Qi J, Wang B etal. A three dimensional electrochemical oxidation reactorbased on magnetic steel slag particle electrodes for printing/dyeing wastewater treatment. Harbin Gongye Daxue Xuebao/Journal of Harbin Institute of Technology, 2015 Aug; 47(8): 38-42.

[14] Wang K, Wang M Q, Lv W, et al. Optimization and assessment on indirect electrochemical reduction of indigo. Pigment and Resin Technology. 2019 Oct; 49(2): 154-162.

[15] Bechtold T, Turcanu A. Electrochemical reduction in vat dyeing: greener chemistry replaces traditional processes. Journal of Cleaner Production. 2009 Dec; 17(18): 1669-1679.

[16] Bechtold T, Turcanu A, Schrott W. Electrochemical reduction of CI sulphur black 1-correlation between electrochemical parameters and colour depth in exhaust dyeing. Journal of Applied Electrochemistry. 2008 Aug; 38(1): 25-30.

[17] Guilherme GB and Juliano CC and Maria VBZ. Enhanced photoelectrocatalytic degradation of an acid dye with boron-doped TiO2 nanotube anodes. Catalysis Today. 2015 Feb; 240: 100-106.

[18] Qin L, et al. One-step construction of Pickering emulsion via commercial TiO2 nanoparticles for photocatalytic dye degradation. Applied Catalysis B: Environmental. 2019 Jul; 249: 1-8.

[19] Firoz Babu K, Senthil Kumar R, Anbu Kulandainathan M, et al. Ferric-oxalate-gluconate based redox mediated electrochemical system for vat dyeing. Journal of Applied Electrochemistry. 2009 Jul; 39(7): 1025-1031.

[20] Sheng D, et al. Improved dyeing of poly-m-phenyleneisophthalamide using cationic dye based on macro-cation dyeing mechanism. Dyes and Pigments. 2019 Apr; 163: 111-117.

[21] Gonzalez V, et al. Ultrasound-enhanced hair dye application for natural dyeing formulations. Ultrasonics Sonochemistry. 2019 Apr; 52: 294-304.

[22] Bechtold $\mathrm{T}$ and Turcanu A. Determination of reaction rate between cathodically formed Fe II triethanolamine-complex and Fe III-hepta-d-gluconate complex by cyclic voltammetry. Journal of Electroanalytical Chemistry. 2005 Jun; 580(1): 173-178. 
[23] Zhe-Jian Y, et al. Thermally activated delayed fluorescence (TADF) dyes as efficient organic photosensitizers for photocatalytic water reduction. Catalysis Communications. 2018 Sep; 119.

[24] Albert R and Xiunan J. State of the art technologies and new electrochemical methods for the reduction of vat dyes. Dyes and Pigments. 2003 Dec; 59(3): 223-235.

[25] Wenjuan L, et al. Novel approach to enhance photosensitized degradation of rhodamine B under visible light irradiation by the ZnxCd1-xS/TiO2 nanocomposites. Environmental Science \& Technology. 2011 Apr; 45(7): 2987-2993.

[26] Socha A, et al. Electrochemical and photoelectrochemical degradation of direct dyes. Coloration Technology. 2006 Jul; 122(4): 207-212.

[27] Pengfei Z, et al. Structure and properties of Ag3PO4 /diatomite photocatalysts for the degradation of organic dyes under visible light irradiation. Powder Technology. 2018 Aug; 336: 230-239.

[28] Bhavani R and Sivasamy A. Sonocatalytic degradation of malachite green oxalate by a semiconductor metal oxide nanocatalyst. Ecotoxicology and Environmental Safety. 2016 Dec; 134: 403-411. 